\section{Epinephrine administration via a laryngeal mask airway: what is the optimal dose?}

\author{
CHIN-KUN LIAO • HUNG-JUNG LIN • NING-PING FOO • \\ CHIN-CHAN LIN • HOW-RAN GUO • KUO-TAI CHEN
}

KUO-TAI CHEN $(\bowtie) \cdot$ HUNG-JUNG LIN

Emergency Department

Chi-Mei Medical Center 901 Chung-Hwa Road, Yung Kang Tainan 710 , Taiwan Phone: 886-6-2812811 ext. 57196 Fax: 886-6-2816161 E-mail:890502@mail.chimei.org.tw

CHIN-KUN LIAO • NING-PING FOO

Department of Emergency Medicine Chi-Mei Medical Center, Liouying

HUNG-JUNG LIN Department of Biotechnology, Southern Tainan University of Technology

CHIN-CHAN LIN Department of Medical Research Chi-Mei Medical Center

HOW-RAN GUO

Department of Environmental and Occupational Health and Department of Environmental and

Occupational Medicine, Medical College,National Cheng Kung University

KUO-TAI CHEN School of Medicine, Taipei Medical University

\begin{abstract}
Background. The aim of this animal study was to clarify the effects of laryngeal mask airway (LMA)-administrated epinephrine and to assess the optimal dose.

Methods. Thirty pigs were anesthetized and intubated with a cuffed tracheal tube (TT) and an LMA. Then they were assigned to one of five groups. The control group received distilled water $10 \mathrm{~mL}$ via the TT; the TT group received epinephrine $50 \mu \mathrm{g} /$ $\mathrm{kg}$ via the TT; and the other three groups received two, four or six times the TT dose of epinephrine via the LMA. Heart rate $(H R)$ and arterial pressure were monitored before and after drug administration for 15 minutes.

Results. After epinephrine administration, the LMA-6 and TT groups had elevated systolic, diastolic and mean arterial pressures at 1 min and there was no significant difference between the two groups. In the TT group, these parameters peaked at 2 min then declined rapidly. In the LMA-6 group, they increased more slowly, and then maintained a plateau. The control, LMA-2 and LMA-4 groups failed to display significant persistent (>2 min) hemodynamic changes.

Conclusions. We could not identify an optimal LMA-administrated epinephrine dose. The TT route is suitable when a high peak drug effect is required and the LMA route may be preferable if a persistent plateau effect is desired. Effective LMA administration of drugs may require larger doses than those given via TT.
\end{abstract}

Keywords: airway, drug delivery, epinephrine, laryngeal mask airway (LMA), tracheal tube

\section{Introduction}

Laryngeal mask airways (LMA) are widely used in anaesthesia and resuscitation. $(1,2)$ They are easy to use and require minimal training, which makes them an excellent alternative airway device for personnel who are not familiar with airway management. (3-5)
Health care workers have been giving drugs through airway devices for decades. (6) Among these devices, the tracheal tube (TT) is most commonly used. However, considerable training is required to acquire and maintain the 
skill of tracheal intubation. (7) Our previous studies revealed that using a catheter inserted through an LMA into the trachea to deliver drugs is as effective as using the TT route. $(8,9)$ However, the ease of passing a catheter and documenting its correct position are still under investigation. $(10,11)$

Trevisanuto et al. and Spain et al. reported that delivering surfactant and bronchodilator via an LMA is effective in treating neonatal respiratory distress syndrome and bronchospasm, respectively. $(12,13)$ Our previous studies showed that LMA-administered epinephrine was well absorbed through the mucosa of the airway but that, compared with the TT route, the same dose produced a lower plasma level. $(8,9)$ The optimal LMA-administered dose of epinephrine is unknown. We therefore gave increasing doses of LMAadministered epinephrine to identify a dose equipotent to a standard TT dose and to clarify the characteristics of drug absorption via this route.

\section{Materials and Methods}

The Animal Care and Users Committee of the Chi-Mei Medical Center approved the study and the animals were cared for in accordance with national and institutional guidelines.

Thirty domestic Yorkshire pigs, nine males and 23 females, weighing 16.5$27 \mathrm{~kg}$ were anesthetized with intravenous pentothal $30 \mathrm{mg} / \mathrm{kg}$ and randomly assigned to one of five groups:

Control Group: $10 \mathrm{~mL}$ of distilled water via the TT.

TT Group: epinephrine $50 \mu \mathrm{g} / \mathrm{kg}$ via the TT.

LMA-2 Group: epinephrine $100 \mu \mathrm{g} / \mathrm{kg}$ via the LMA (twice the TT group dose). LMA-4 Group: epinephrine $200 \mu \mathrm{g} / \mathrm{kg}$ via the LMA (four times the TT group dose).

LMA-6 Group: epinephrine $300 \mu \mathrm{g} / \mathrm{kg}$ via the LMA (six times the TT group dose).

All doses of epinephrine were diluted to a total volume of $10 \mathrm{~mL}$ with distilled water. The animals were intubated with a cuffed TT (internal diameter 5 $\mathrm{mm}$ ) and size 3 LMA (LMA-Class ${ }^{T M}$,

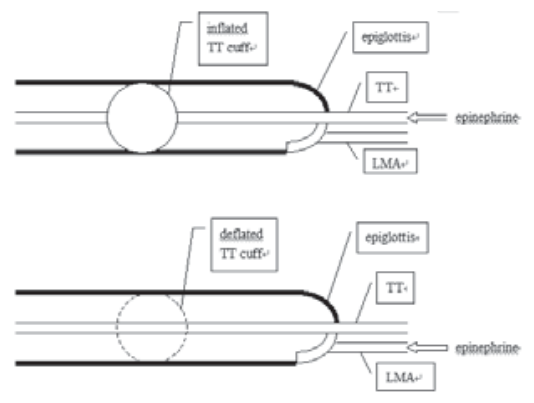

LMA, laryngeal mask airway; TT, tracheal tube.

Figure 1. In the control and TT groups, epinephrine was given via the TT (upper picture). In the LMA-2, LMA-4 and LMA-6 groups, epinephrine was given via the LMA (lower picture).

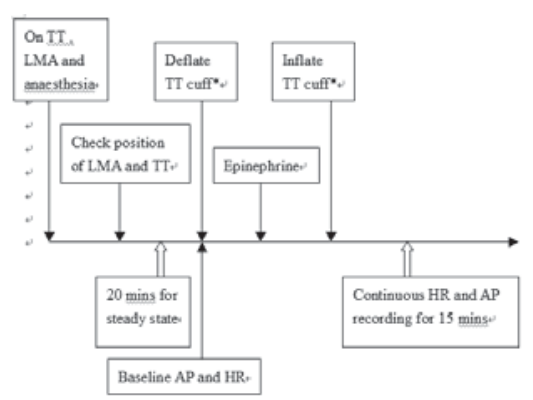

$A P$, arterial pressure; $H R$, heart rate; LMA, laryngeal mask airway; TT, tracheal tube.

Figure 2. Timeline for the experimental protocol. *Inflate and deflate TT cuff in the LMA-2, LMA-4 and LMA-6 groups.

Laryngeal Mask Company, Henley on Thames, UK) (figure 1). We used a fibre-optic bronchoscope to confirm the position of the TT and to ensure that the opening of the larynx faced directly towards the lower aperture of the LMA. The animals were ventilated via the TT with a respiratory rate of 15 breath/min, tidal volume $20 \mathrm{~mL} / \mathrm{kg}$ and $\mathrm{FiO}_{2}$ 1.0. All

\section{Table 1. Biometric data.}

\begin{tabular}{llllll}
\hline Group & Control & TT & LMA-2 & LMA-4 & LMA-6 \\
\hline $\begin{array}{l}\text { Number of animals } \\
(\mathrm{M} / \mathrm{F})\end{array}$ & $6(2 / 4)$ & $6(2 / 4)$ & $6(3 / 3)$ & $6(1 / 5)$ & $6(1 / 5)$ \\
\hline
\end{tabular}

Weight $(\mathrm{kg}$, mean $\pm \mathrm{SD})$ $21.6 \pm 3.5 \quad 21.1 \pm 3.0 \quad 22.9 \pm 3.1 \quad 21.2 \pm 2.3 \quad 21.6 \pm 2.2$

LMA, laryngeal mask airway; TT, tracheal tube. animals were attached to a three-lead ECG monitor and given an infusion of $0.9 \%$ normal saline solution at $20 \mathrm{~mL} / \mathrm{h}$ throughout the experiment. A carotid or femoral catheter was inserted for continuous heart rate $(\mathrm{HR})$ and arterial blood pressure (BP) monitoring. An intravenous flush of heparin $3000 \mathrm{IU}$ was given to avoid intra-catheter clot formation.

To achieve steady state conditions, a 20-min stabilization period followed the completion of the above procedures. We recorded baseline HR and BP. Subsequently, we deflated the cuff of the TT in the LMA-2, LMA-4 and LMA-6 groups to facilitate spraying epinephrine into the trachea through the LMA. In the control and TT groups, epinephrine and distilled water were delivered via the TT and in the other groups via the LMA. Drug administration was followed by five forceful artificial respirations using an Ambu Bag. The cuff of the TT was re-inflated after epinephrine administration in the LMA groups. HR and BP were recorded continuously for $15 \mathrm{~min}$ following medication delivery. At the end of the experiment, the animals were killed by injection of potassium chloride solution $10 \mathrm{mEq}$. The methods of medication administration are presented in figure 1; all other procedures are illustrated in figure 2 .

All experimental results are presented as medians unless otherwise noted (table 1).

The differences in HR and BP between the five groups were compared using the Kruskal-Wallis followed by MannWhitney $U$ tests. The intra-animal changes in hemodynamic variables were investigated using the Wilcoxon signed rank test. All statistical tests were performed at a two-tailed significance level of 0.05 . 


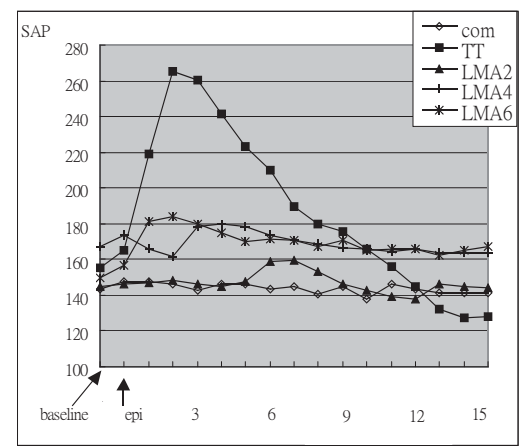

LMA, laryngeal mask airway; TT, tracheal tube.

Figure 3. Systolic arterial pressure (SAP) changes following epinephrine administration.

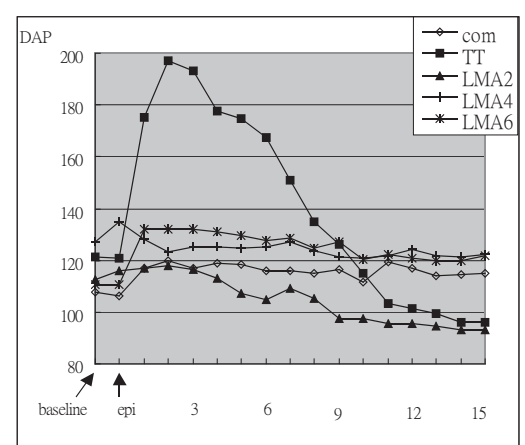

LMA, laryngeal mask airway; TT, tracheal tube.

Figure 4. Diastolic arterial pressure (DAP) changes following epinephrine administration.

\section{Results}

There were no significant differences in biometric data between the five groups (table 1). The hemodynamic changes following epinephrine administration via the TT or LMA are illustrated in figures 3-6.

The LMA-6 and TT groups had an increase in systolic arterial pressure (SAP) at 1 min post-injection $(p=0.028$, both groups). The response lasted for $11 \mathrm{~min}$ and $8 \mathrm{~min}$, respectively. The TT group had the highest peak SAP, at 2 min post-injection. However, the increase in SAP lasted longer in the LMA-6 group. There was no significant difference between the two groups $(p=0.605)$.

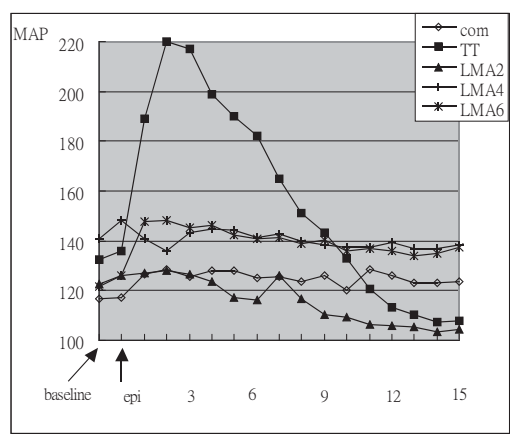

LMA, laryngeal mask airway; TT, tracheal tube.

Figure 5. Mean arterial pressure (MAP) changes following epinephrine administration.

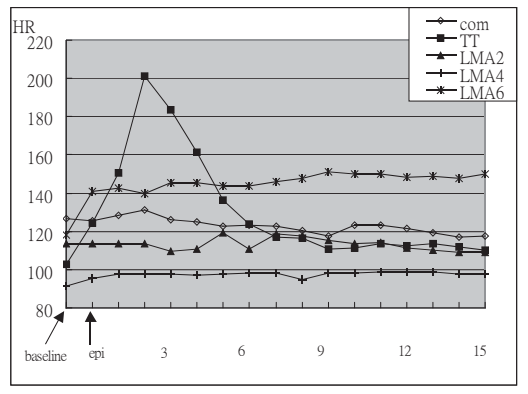

LMA, laryngeal mask airway; TT, tracheal tube.

Figure 6. Heart rate (HR) changes following epinephrine administration.

Both the LMA-6 and TT groups had significant elevations in diastolic arterial pressure (DAP) 1-7 min and in mean arterial pressure (MAP) 1-8 min postinjection, respectively. Similarly, the TT group reached the highest peak DAP and MAP at 2 min after epinephrine administration. There was no significant difference between the groups $(p=0.629$ in DAP and $p=0.642$ in MAP). The HR increased in the TT group at 0.5 min and in the LMA- 6 group at 1 min post-injection $(p=0.028$ and $p=0.046$, respectively). The effect persisted for 15 min in both groups. The TT group had the highest $\mathrm{HR}$ at $2 \mathrm{~min}$. There was a significant difference between the two groups $(p=0.01)$.
The control, LMA- and LMA-4 groups failed to display significant persistent (>2 min) hemodynamic changes after epinephrine administration.

\section{Discussion}

We tested increasing doses of LMAadministrated epinephrine and compared the effect with the standard tracheal route. After epinephrine administration, both the LMA- 6 and TT groups had significant elevations in SAP, DAP, and MAP. Though there were no statistically significant differences between these two groups, the pattern of hemodynamic changes were distinct. In the TT group, the hemodynamic parameters peaked promptly after epinephrine administration, and then declined rapidly. The peak levels were higher than in the other groups. Those in the LMA-6 group increased more slowly and reached a plateau later. The differences in response presumably reflect different speeds of drug absorption, which are themselves the result of differences in the available area for absorption. With the LMA route, drug absorption occurs partly through the laryngeal mucosa and partly through the broncho-alveolar mucosa. With the TT route, all drug absorption is through the broncho-alveolar mucosa.

Niemann et al. reported that epinephrine given at standard doses via a tracheal tube during cardiac arrest and cardiopulmonary resuscitation (CPR) was of no benefit. (14) European Resuscitation Council CPR guidelines recommend epinephrine delivery via tracheal tube only as the last resort. (15) The onset and the peak pharmacological effect of the LMA-6 group were slower and lower than in the TT group, and cardiac arrest is likely to further decrease drug effects. Therefore, unless higher than six times the tracheal dose is given, it is impractical to administer epinephrine via the LMA in resuscitation scenarios.

Trevisanuto et al. successfully treated neonatal respiratory distress syndrome by LMA-administered surfactant in eight preterm infants. (12) The LMA route avoided the potential risks of tra- 
cheal intubation, such as upper airway damage, infection, bradycardia and potentially catastrophic tracheal malpositioning. For certain drugs where a steady plateau effect instead of a high peak level is desired, the LMA is still an alternative route for drug delivery. However, given the smaller area of drug absorption for the LMA route, doses higher than those recommended for tracheal administration are likely to be required.

There were a number of limitations to our study. First, the LMA fitted loosely in the pig larynx and leakage of epine- phrine solution may have occurred, compromising interpretation of the drug effect. In addition, there was a TT in the trachea: although we deflated the cuff to reduce occlusion, the TT would have impeded dispersion of epinephrine into the broncho-alveolar area and may have led to an underestimate of the effects of LMA-administered epinephrine. Moreover, drug delivery via different airway devices involves different degrees of experimental stress, which may have led to variable changes of heart rate and arterial pressure.

\section{Conclusions}

In comparison with standard tracheal route, LMA administration of six times the standard tracheal dose of epinephrine achieved statistically equivalent hemodynamic changes. The effects achieved via the tracheal route peaked sooner but swiftly declined; those of LMA route rose more slowly and were maintained at a plateau. We could not identify an optimal LMA-administered epinephrine dose. Doses larger than those recommended for tracheal administration may be needed for drugs given via the LMA.

\section{REFERENCES}

1.Pollack Jr CV. The laryngeal mask airway: a comprehensive review for the Emergency Physicians. J Emerg Med 2001;20:53-66.

2.Verghese C, Brimacombe JR. Survey of laryngeal mask airway usage in 11,910 patients: safety and efficacy for conventional and nonconventional usage. Anesth Analg 1996;82:129-33.

3.Davies PR, Tighe SQ, Greenslade GL, Evans GHI. Laryngeal mask airway and tracheal tube insertion by unskilled personnel. Lancet 1990;336:977-9

4.Dickinson M, Curry P. Training for the use of the laryngeal mask in emergency and resuscitation situations. Resuscitation 1994;28:111-3.

5.Baskett PJF. The use of the laryngeal mask airway by nurses during cardiopulmonary resuscitation. Results of a multicentre trial. Anaesthesia 1994;49:3-7.

6.Greenberg Ml. The use of endotracheal medication in cardiac emergencies. Resuscitation 1984;12:155-6.

7.Sayre MR, Sakles JC, Mistler AF, Evans JL, Kramer AT, Pancioli AM. Field trial of endotracheal intubation by basic EMTs. Ann Emerg Med 1998;31:228-33

8.Chen KT, Lin HJ, Guo HR, Lin NT, Lin CC. Feasibility study of epinephrine administration via laryngeal mask airway using a porcine model. Resuscitation 2006;69:503-7.

9. Chen KT, Lin HJ, Jeng HW, Lin CC, Guo HR. The pharmacological effect of epinephrine administration via laryngeal mask airway in a porcine model of asphyxial cardiac arrest. Emerg Med J 2008;25:722-4.

10.Alexander R, Swales H, Pickford A, Smith GB. The laryngeal mask airway and the tracheal route for drug administration. Br J Anaesth 1997;78:220-1.

11.Liao CK, Lin HJ, Chen KT. An easy method to administer drugs into the trachea via the intubating laryngeal mask airway. Am J Emerg Med 2008;26:370-1.

12. Trevisanuto D, Grazzina N, Ferrarese P, Micaglio M, Verghese C, Zanardo V. Laryngeal mask airway used as a delivery conduit for the administration of surfactant to premature infants with respiratory distress syndrome. Biol Neonate 2005;87:217-20.

13.Spain BT, Riley RH. Salbutamol via the laryngeal mask for relief of bronchospasm. Anaesthesia 1992;47:1107.

14.Niemann JT, Stratton SJ, Cruz B, Lewis RJ. Endotracheal drug administration during out-of-hospital resuscitation: where are the survivors. Resuscitation 2002;53:153-7.

15.Nolan JP, Deakin CD, Soar J, Bottiger BW, Smith G; European Resuscitation Council. European Resuscitation Council guidelines for resuscitation 2005. Section 4. Adult advanced life support. Resuscitation 2005;67 Suppl 1:S39-86. 